\title{
Impact of an Online Education Program on Counselors' Knowledge and Attitudes About Near-Death Experiences
}

\author{
Saharnaz Loseu, PhD, LPC-Intern, NCC, \\ and Janice Miner Holden, EdD, LPC-S, NCC, ACMHP \\ University of North Texas
}

\begin{abstract}
An estimated 15 million people in the US have had a near-death experience (NDE) involving usually lucid consciousness during a close brush with death. They have reported psychologically harmful experiences disclosing their NDEs to healthcare professionals, including counselors. Counselors' knowledge and attitudes about NDEs appear to be critical variables in their ability to respond therapeutically to clients' NDE disclosures. The recent development of a psychometrically sound instrument to assess these variables, coupled with online availability of a three-part NDE educational program for health professionals, made possible for the first time a large-scale pre-post study of the effect of the program on counselors' knowledge and attitudes about NDEs. Participants were 212 licensed professional counselors (LPCs) aged 23 to 71 years old $(M=$ 44.93, $S D=12.69$ ); sex self-identified as $12.3 \%$ male, $87.3 \%$ female, and .5\% other; race/ethnicity self-identified as $84 \%$ White and $17 \%$ non-White and as 6.6\% Latino/Hispanic and 92.5\% non-Latino/Hispanic; and representing four regions of the US. Compared to a control group composed of LPCs who completed topically unrelated online programs $(n=112)$, those who completed the NDE program $(n=100)$ showed significantly more accurate knowledge and more positive attitudes about NDEs. Participants also overwhelmingly expressed enjoyment of the programs. These results support the use of online training to increase counselors' knowledge and improve their attitudes about NDEs so they
\end{abstract}

Saharnaz Loseu, PhD, LPC-Intern, NCC, completed her doctoral degree in Counseling at the University of North Texas (UNT), Denton, TX. Her clinical interests are transpersonal concerns in counseling, grief, and trauma. She wishes to thank the UNT College of Education for Dissertation and Thesis Support Funding and the Department of Counseling \& Higher Education for the Transpersonal Counseling Dissertation Scholarship, both of which supported her dissertation research upon which this article is based. Janice Miner Holden, EdD, LPC-S, NCC, ACMHP, is professor of Counseling and chair of the Department of Counseling and Higher Education at UNT in Denton, TX. Her primary research interest is the transpersonal perspective in counseling with an emphasis on near-death experiences. Correspondence regarding this article should be sent to Dr. Loseu at email: sahar.loseu@gmail.com. 
can provide clinically and ethically sound treatment to near-death experiencers. Limitations of the study and future research are addressed.

KEY WORDS: near-death experiences; counselors; knowledge and attitude; NDE education; online education

Out of all people who survive a close brush with death, approximately $80 \%$ do not recall anything unusual; the other approximately $20 \%$ recall a unique experience of usually lucid consciousness: a near-death experience (NDE; Holden, 2017). Although scholars have addressed NDEs since at least the end of the 19th century (Sidgwick, 1885), in the late 20th century Raymond Moody (1975) coined the term near-death experience, and since then, various scholars have further enhanced the definition of NDEs (Greyson, 2014). Scholars have characterized NDEs as experiences that typically occur during close brushes with death precipitated by illness, injury, or attempted suicide (Zingrone \& Alvarado, 2009). A unique subjective characteristic of near-death experiencers (NDErs) is that they typically experience their consciousness functioning separately from their bodies, perceiving the material world and/or transmaterial environments and entities such as deceased loved ones and/or spiritual or religious figures (Zingrone \& Alvarado, 2009). Bruce Greyson (2000), a prominent NDE researcher, defined NDEs as "profound psychological events with transcendental and mystical elements, typically occurring to individuals close to death or in situations of intense physical or emotional danger" (pp. 315-316).

NDEs are common in a variety of populations and occur regardless of age, sex, sexual orientation, religious affiliation, socioeconomic class, mental health status, and ethnic group (Fenwick \& Fenwick, 1995; Holden, 2012; Holden, Long, \& MacLurg, 2009). Furthermore, NDEs are prevalent. The results of more than 40 years of research on the phenomena revealed that more than 15 million Americans have had an NDE (Taylor \& van Lommel, 2006). NDEs not only are a common experience but also share similar content.

The content of NDEs can be predominantly either pleasurable or distressing. Whereas pleasurable NDEs carry an overall positive emotional tone of peace, love, and/or bliss (Zingrone \& Alvarado, 2009), distressing NDEs carry a tone of panic, guilt, loneliness, and/or fear (Bush, 2009). Pleasurable NDEs account for approximately $90 \%$ of reported experiences (Holden, 2017). Both types of NDEs can have psychological, spiritual, physical, and social aftereffects (Greyson, 1997; 
Greyson, Kelly, \& Kelly, 2009; Holden, 2012; Noyes, Fenwick, Holden, \& Christian, 2009). NDEs vary in their depth-the presence and/or intensity of various elements such as perceiving light, meeting spiritual entities, and experiencing a life review; in general, the deeper the NDE, the more numerous and intense the aftereffects (Noyes et al., 2009).

Life changing and often stressful aftereffects of NDEs propel many NDErs to seek support for the integration of their experiences (Greyson, 2000). Often, healthcare professionals are NDErs' first confidants, and their reactions can influence NDErs' subsequent integration of the experience into their daily lives (Foster, James, \& Holden, 2009; Greyson, 1997; Groth-Marnat \& Summers, 1998). In particular, the initial NDE disclosure experience typically plays an important role in subsequent integration of the NDE (Holden, 2012; Noyes et al., 2009).

Initially, many healthcare providers viewed NDEs as hallucinations or as evidence of psychological illness-perspectives that research has since shown to be inaccurate (Greyson, 1997). Compared to healthcare providers who are knowledgeable about NDEs, those who are not knowledgeable may be more prone to reject, pathologize, or discount NDEs. When NDErs face a positive and supportive attitude during their disclosures, they internalize and integrate their NDEs more positively than when they face rejection (Greyson \& Liester, 2004). Consequently, negative disclosure experiences can be harmful to NDErs. In a quantitative study of 88 NDErs' perceptions of 188 of their NDE disclosure experiences, they "considered 19\% of their most noteworthy disclosure experiences to healthcare professionals to have ranged from mildly to extremely negative, unpleasant, and harmful" (Holden, Kinsey, \& Moore, 2014, p. 284). NDErs identified counselors among the healthcare professionals with whom they had negative disclosure experiences.

Counselors abide by the ethical principle of nonmaleficence (Herlihy \& Corey, 2006, p. 9), or avoiding intentional or unintentional infliction of harm on their clients (American Counseling Association [ACA], 2014; Forester-Miller \& Davis, 1995). Counselors' knowledge and attitudes about NDEs appear to be critical variables in their ability to uphold the ethical imperative to do no harm to NDEr clients. However, some counseling professionals and students possess inaccurate knowledge about and negative attitudes about NDEs (Pace, 2013), and the disclosure experiences of NDErs whose experiences occurred over 
seven decades did not improve over time (Holden et al., 2014). Thus, the availability of increased empirically based information regarding NDEs in recent years has not translated into increased knowledge on the part of counselors or improved disclosure experiences on the part of NDEr clients. These results point to a need for more targeted educational programs for counselors in order to increase their knowledge and improve their attitudes about NDEs and, thus, hopefully to reduce or eliminate harmful disclosure experiences for NDErs.

Although some health professionals have designed NDE-related curricula and informally observed them to be effective (McEvoy, 1990; Sheeler, 2005), only one study to date used quantitative measures to assess effectiveness of an NDE educational curriculum. Undergraduate psychology students completed a pretest about their knowledge of NDEs, watched and discussed an hour-long documentary about them (Broome, 2002), then completed a post-test. Results revealed a significant increase in accurate knowledge (Holden, Oden, Kozlowski, \& Hayslip, 2011). Authors representing a variety of healthcare preparation professions have repeatedly urged educators to include NDErelated information in their curricula, but to date such inclusion has been very much the exception rather than the rule (Foster, James, \& Holden, 2009). One challenge has been the availability of concise, high-quality material; another is access to a wide-reaching delivery method. An additional challenge for researchers of curriculum effectiveness has been the availability of a psychometrically sound instrument to assess knowledge and attitudes about NDEs.

Regarding the delivery method challenge, one promising approach is online education. Although this method has drawbacks, such as challenges in increasing student engagement, developing effective methods of learning and assessment, and utilizing cost effective technology (Anderson, 2008), it has numerous advantages: It is more available than face-to-face education and is self-paced, convenient, and remotely accessible. Additionally, online education is not only appropriate but also considered as effective as traditional classroom education (Benshoff \& Gibbons, 2011; Dominguez \& Ridley, 2001; Zhao, Lei, Yan, Lai, \& Tan, 2005). Moreover, student satisfaction and performance in an online learning environment is comparable with satisfaction and performance in face-to-face environments (Blackmore, Tantam, \& van Deurzen, 2006; Blackmore, van Deurzen, \& Tantam, 2005). More importantly, counselor educators have integrated online education into counselor education programs-including those accredited by the Council for Accreditation of Counseling and Related Educa- 
tional Programs (CACREP), and many counseling professionals currently utilize this learning modality (Lewis \& Coursol, 2007).

Regarding availability of concise, high-quality material, reputable sources have recently developed online curricula addressing NDEs (Holden, 2008; Holden, 2015; Roberta Moore Video Productions, 2013). Regarding the final challenge of assessment, the recently developed Knowledge and Attitudes About Near-Death Experiences Scale (KANDES; Pace, Holden, Blalock, Holliman, \& Henson, 2016) is a valid and reliable instrument to measure the effects of interventions on knowledge and attitudes about NDEs.

Having now the means to investigate, we pursued the following question:

1. Does an online education program about NDEs increase knowledge and improve attitudes about NDEs among LPCs who receive the intervention in comparison to LPCs who receive an online education program about non-NDE topics?

a) What is the direction and magnitude of the relationship between LPCs' knowledge and attitudes about NDEs?

\section{Methods}

\section{Participants}

LPCs from across the US who were fully and actively licensed in their states as determined by their state licensing boards represented the population of interest for this study. Ideally, every individual in the population of interest would participate in the study to minimize the generalizability limitations of the study; however, due to lack of email access to LPCs nationwide, we used a convenience sample comprised of LPCs in states in which their licensing boards provided access to their email addresses either for a cost or free of cost. We recruited participants from one state selected from each of the four ACA (2016) regions: Ohio (Midwest), Rhode Island (North Atlantic), Florida (Southern), and Wyoming (Western). A total of 18,088 LPCs received an electronic invitation to participate; the invitation contained a randomly assigned link to either the experimental or the control prong of the study. We incentivized participation with two National Board for Certified Counselors (NBCC) continuing education credits for those who completed all study requirements. Additionally, the first 100 participants received a $\$ 25$ Visa gift card. We sent two email reminders, and the research assistants made 50 total phone calls-until we 
achieved a target number of experimental-group completers sufficient for statistical power to conduct analyses. Of the $498 \mathrm{LPCs}$ who began the experimental condition, 100 completed it, and of the 536 LPCs who began the control condition, 112 completed it; these 212 completers represented a $20.5 \%$ completion rate and a $1.36 \%$ response rate. See Table 1 for participant demographics.

\section{Instrumentation}

Participants demonstrated their knowledge and attitudes about NDEs through their scores on the KANDES, a 46-item, Likert-type scale with two subscales: Knowledge and Attitude (Pace et al., 2016). The Knowledge subscale contains 23 items, each with 5 response choices ranging from Completely True to Completely False. The Attitude subscale contains 23 items, each with 7 response choices ranging from Completely Disagree to Completely Agree. Examples of items from the Knowledge subscale are: Near-death experiencers (NDErs) frequently report feeling a deep sense of fear when encountering the light during their experience, Most people who experience NDEs are profoundly changed for decades after the experience, and Individuals' values before an NDE are usually compatible with their values after their experience. Examples of items from the Attitude subscale are: I would question my client's mental health for having had the experience. I would not doubt my client's sanity just for having had the experience, and I would think that my client's experience was purely imaginary-like a dream or hallucination. For the Knowledge and Attitude subscales, respectively, the KANDES contains both accurate/positively- and inaccurate/negatively-worded items; negatively worded items were reverse scored so that disagreement indicated a positive response. A higher total score indicated more accurate knowledge or more positive attitudes. The KANDES takes approximately 10 minutes to complete.

Pace et al. (2016) concluded that the KANDES meets psychometric standards for reliability and validity. Holden, Fekken, and Cotton (1991) asserted that acceptable Cronbach's alpha scores are .70 or greater. Pace et al. (2016) found Cronbach's alpha scores for the Knowledge and Attitude subscales were .816 and .909, respectively, indicating strong inter-item correlation and internal consistency. For test-retest, Pearson's $r$ correlation coefficients closer to 1.0 demonstrate stronger relationship between the variables (Tabachnick \& Fidell, 2007). Pearson's $r$ for the Knowledge and Attitude subscales were .812 and .748, respectively, indicating good consistency (Landis 


\begin{tabular}{|c|c|c|c|}
\hline \multirow[b]{2}{*}{ Variable } & \multicolumn{3}{|c|}{$n(\%)$ or Mean (SD) } \\
\hline & $\begin{array}{c}\text { Total } \\
(N=212)\end{array}$ & $\begin{array}{l}\text { Experi- } \\
\text { mental } \\
(n=100)\end{array}$ & $\begin{array}{c}\text { Control } \\
(n=112)\end{array}$ \\
\hline \multicolumn{4}{|l|}{ Years Held Licensure } \\
\hline Mean (SD) & $9.78(8.5)$ & $9.84(8.9)$ & $9.73(8.1)$ \\
\hline Range & $1 \mathrm{Mo}-39$ Yrs & $1-37$ Yrs & $1 \mathrm{Mo}-39$ Yrs \\
\hline \multicolumn{4}{|l|}{ Age (Years) } \\
\hline Mean (SD) & $44.93(12.7)$ & $45.92(12.5)$ & $44.12(12.9)$ \\
\hline Range & $23-71$ & $26-71$ & $23-71$ \\
\hline \multicolumn{4}{|l|}{ Sex } \\
\hline Male & 26 (12.3\%) & $13(13 \%)$ & $13(11.6 \%)$ \\
\hline Female & $185(87.3 \%)$ & $87(87 \%)$ & $98(87.5 \%)$ \\
\hline Other & $1(0.5 \%)$ & $0(0.0 \%)$ & $1(0.9 \%)$ \\
\hline \multicolumn{4}{|l|}{ Education Level } \\
\hline Master's Degree & $186(87.7 \%)$ & $84(84.0 \%)$ & $102(91.1 \%)$ \\
\hline Doctoral Degree & $18(8.5 \%)$ & $12(12.0 \%)$ & $6(5.4 \%)$ \\
\hline Other & $8(3.8 \%)$ & $4(4.0 \%)$ & $4(3.6 \%)$ \\
\hline \multicolumn{4}{|l|}{ Racial Group } \\
\hline Black/African American & $18(8.5 \%)$ & $6(6.0 \%)$ & $12(10.7 \%)$ \\
\hline Asian & $2(0.9 \%)$ & $1(1.0 \%)$ & $1(0.9 \%)$ \\
\hline White/Caucasian & $176(83.0 \%)$ & $85(85.0 \%)$ & $91(81.3 \%)$ \\
\hline Native American & $1(0.5 \%)$ & $1(1.0 \%)$ & $0(0.0 \%)$ \\
\hline Multiracial & $8(3.8 \%)$ & $3(3.0 \%)$ & $5(4.5 \%)$ \\
\hline Other & $7(3.3 \%)$ & $4(4.0 \%)$ & $3(2.7 \%)$ \\
\hline \multicolumn{4}{|l|}{ Latino/Hispanic } \\
\hline Yes & $14(6.6 \%)$ & $7(7.0 \%)$ & $7(6.3 \%)$ \\
\hline No & $196(92.5 \%)$ & $92(92.0 \%)$ & $104(92.9 \%)$ \\
\hline \multicolumn{4}{|l|}{ Exposure to STEs } \\
\hline NDEs & $77(36.3 \%)$ & $42(42.0 \%)$ & $35(31.4 \%)$ \\
\hline After-Death Communication & $52(24.5 \%)$ & $30(30.0 \%)$ & $22(19.6 \%)$ \\
\hline Past Life Memories & $36(17.0 \%)$ & $18(18.0 \%)$ & $18(16.1 \%)$ \\
\hline \multicolumn{4}{|l|}{ Previous NDE Exposure Through } \\
\hline Graduate School Training & $4(1.9 \%)$ & $3(3.0 \%)$ & $1(0.9 \%)$ \\
\hline Clients' NDE & $45(21.2 \%)$ & $16(16 \%)$ & $29(25.9 \%)$ \\
\hline Friends'/Relatives'/Self's Own NDE & $50(23.6 \%)$ & $21(21 \%)$ & $29(25.9 \%)$ \\
\hline \multicolumn{4}{|l|}{ Religious/Spiritual Affiliation } \\
\hline None/Atheist & $21(9.9 \%)$ & $10(10.0 \%)$ & $11(9.8 \%)$ \\
\hline Christian-Catholic & $40(18.9 \%)$ & $22(22.0 \%)$ & $18(16.1 \%)$ \\
\hline Christian-Protestant & $77(36.3 \%)$ & $31(31.0 \%)$ & $46(41.1 \%)$ \\
\hline Jewish & $5(2.4 \%)$ & $2(2.0 \%)$ & $3(2.7 \%)$ \\
\hline Buddhist & $3(1.4 \%)$ & $3(3.0 \%)$ & $0(0.0 \%)$ \\
\hline Unitarian Universalist & $2(0.9 \%)$ & $1(1.0 \%)$ & $1(0.9 \%)$ \\
\hline Spiritual, not religious & $48(22.6 \%)$ & $23(23.0 \%)$ & $25(22.3 \%)$ \\
\hline Other & $16(7.5 \%)$ & $8(8.0 \%)$ & $8(7.1 \%)$ \\
\hline \multicolumn{4}{|l|}{ Spirituality/Religion } \\
\hline Conservative & $38(17.9 \%)$ & $18(18.0 \%)$ & $20(17.9 \%)$ \\
\hline Moderate & $61(28.8 \%)$ & $26(26.0 \%)$ & $35(31.3 \%)$ \\
\hline Liberal & $92(43.4 \%)$ & $48(48.0 \%)$ & $44(39.3 \%)$ \\
\hline Other & $21(9.9 \%)$ & $8(8.0 \%)$ & $13(11.6 \%)$ \\
\hline
\end{tabular}


\& Koch, 1977). Pace et al. (2016) established validity for the Knowledge subscale by meeting "criteria that emerged from a thorough review of extensive literature on" NDEs (p. 181), and exploratory factor analysis revealed an acceptable amount of variance to substantiate construct validity for the Attitude subscale. For the current sample, the pre-/post-test Cronbach's alphas were, respectively, .85 and .90 for Knowledge and .87 and .91 for Attitude.

\section{Procedures}

Prior to participant recruitment, we obtained Human Subjects approval from the University of North Texas Institutional Review Board. We utilized Qualtrics as the online survey software to launch the components of this study: (a) a consent form, (b) a pretest survey, (c) an intervention, and (d) a posttest survey. Each participant received a personalized email with a brief description of the study-referring only to the effect of online counselor education instruction rather than to the specific intervention topics, information related to the incentive, and a randomly assigned link to either the control or the experimental prong of the study.

Both groups completed the pretest, consisting of demographic questions and the KANDES, and the posttest, consisting of the KANDES and additional questions related to program evaluation for continuing education purposes. The experimental group's intervention consisted of three online components: (a) viewing the 29-minute video Near-Death Experience: What Medical Professionals Need to Know (Roberta Moore Video Productions, 2013); (b) completing the 75-minute interactive program Near-Death Experiences, Part 1: Recognizing a Pleasurable NDE (Holden, 2008); and (c) viewing the 38-minute PowerPoint-with-audio presentation Avoiding Harm: Responding Therapeutically to Disclosure of a Near-Death Experience (Holden, 2015). The control group's intervention consisted of two components: viewing PowerPoint-withaudio programs on the topics of spirituality in counseling (68 minutes) and counseling for process addictions (70 minutes). The survey was designed so that participants could complete the posttest KANDES only after the necessary time had elapsed to complete the educational material. Duration of the study was approximately 2.75 hours for both groups. Participants had the option to complete the study in multiple sittings.

Upon each participant's completion of the post-test, Qualtrics displayed a designated email address to which participants were invited 
to send their name and email address to receive their continuing education certificate and, if applicable, their mailing address to receive a gift card. This step ensured that participant information remained separate from study responses. Within 72 hours of receiving a request, we completed a continuing education certificate and emailed it to the participant as a pdf document attached to a reply email and, if applicable, mailed a gift card.

\section{Data Analysis}

The primary research question in the study was rooted in finding group differences. One method of identifying group differences or membership from a set of outcome variables is to utilize discriminant analysis (Tabachnick \& Fidell, 2007). Kim and Sherry (2010) asserted that descriptive discriminant analysis (DDA) "can examine the most parsimonious way to discriminate between groups, investigate the amount of variance accounted for by the discriminant variables, and evaluate the relative contribution of each discriminant (continuous) variable in classifying the groups" (p. 2). Thus, we utilized DDA to answer the primary research question in this study. Additionally, DDA was the analysis that best fit the research question because it minimized Type I error in lieu of conducting a series of ANOVAs to determine group differences (Sherry, 2006). Finally, DDA honored the relational intricacies of research variables (Sherry, 2006). DDA's sensitivity to relationship between variables was mirrored in the hypothesis that knowledge and attitudes about NDEs may be related in a meaningful way. Therefore, based on the nature of the research question, DDA was an optimal analysis to answer the primary research question.

We set statistical significance level at $p<.05$. We used canonical discriminant function to determine statistical significance by creating a synthetic variable from combining the discriminating variables (Sherry, 2006). In the primary research question, the discriminating variables of interest were knowledge and attitude. To identify if there was a significant difference between the experimental and control groups on Knowledge and Attitude subscales, we examined the canonical function by attending to Wilks's Lambda. Smaller lambda coefficients suggested that knowledge and attitudes differentiate better between experimental and control groups (Betz, 1987). Wilks's lambda is the measure of unexplained variance, and effect size is the explained variance. Sherry (2006) noted that 1-Wilks's lambda provides information regarding the magnitude of the effect-analogous 
to eta squared. Watson (2016) suggested an eta squared effect can be measured at small (.02), medium (.13), and large (.26). Additionally, in a meta-analysis of effectiveness of online programs, Means, Toyama, Murphy, Bakia, and Jones (2009) found the mean effect size for 51 studies to be 24 .

Through the secondary research question, we sought to understand the direction and magnitude of the relationship between counselors' knowledge and attitudes about NDEs. We conducted Pearson's product-moment correlation coefficient ( $r$ ) to answer this question. According to Evans (1996), a Pearson's correlation coefficient of .0 - .1 is very weak, .2- .39 is weak, .4-.59 is moderate, .6- .7 is strong, and $.8-1.0$ is very strong. In the absence of norms for effect size in research of the type described in this article, we used Jacob Cohen's (1988) cautious specifications to assess strength of the effect as $r 2=$ at least .1 for small, .3 for medium, and .5 for large effect.

\section{Results}

We conducted the analysis of data for this study using the Statistical Package for the Social Sciences (SPSS) Version 22. We conducted an exploratory data analysis to assess the missingness and normality of the data. We used SPSS software to conduct data manipulation procedures such as reverse coding for the negatively worded items and computing the KANDES responses. We reported univariate statistics (e.g., means and standard deviations).

This study required a comparison of participants' knowledge and attitudes prior to educational intervention and following educational intervention. DDA addresses between-subject and not within-subject independent variables. Thus, we calculated the difference between pre- and post-test Knowledge scores, which we termed the knowledge variable, and the difference between pre- and post-test Attitude scores, which we termed the attitude variable. Means and standard deviations for intervention and control groups on Knowledge and Attitude subscales appear in Table 2. An independent-samples $t$-test to compare participants' knowledge and attitudes in the two groups at pretest revealed no significant differences: for knowledge, control $(M=86.9, S D=9.4)$ and experimental $(M=85.9, S D=8.7), t(210)=.8$, $p=.422$; for attitude, control $(M=138.7, S D=13.9)$ and experimental $(M=137.9, S D=15.6) ; t(210)=.39, p=.70$.

Accurate interpretation of DDA results are contingent on several assumptions about data (Klecka, 1980; Sherry, 2006; Tabachnick \& Fidell, 2007). These assumptions are: 
Table 2 KANDES Score Means and Standard Deviations at Pre-test and Post-test

\begin{tabular}{|c|c|c|c|c|}
\hline & \multicolumn{2}{|c|}{ Pretest } & \multicolumn{2}{|c|}{ Posttest } \\
\hline & $M$ & SD & $M$ & SD \\
\hline \multicolumn{5}{|c|}{$\begin{array}{l}\text { Experimental Group } \\
(n=100)\end{array}$} \\
\hline Knowledge & 85.9 & 8.7 & 100.4 & 10.1 \\
\hline Attitude & 137.9 & 15.6 & 149.8 & 16.1 \\
\hline \multicolumn{5}{|c|}{$\begin{array}{l}\text { Control Group } \\
(n=112)\end{array}$} \\
\hline Knowledge & 86.9 & 9.4 & 88.1 & 10.4 \\
\hline Attitude & 138.7 & 13.9 & 141.4 & 14.4 \\
\hline
\end{tabular}

(a) availability of two or more mutually exclusive groups, (b) a minimum of two subjects per group, (c) any number of continuous variables as long as the sample size of the smallest group exceeds the number of continuous variables, (d) continuous variables are measured at the interval level, (e) no continuous variable may be a linear combination of other continuous variables, (f) each group must demonstrate multivariate normal distribution on the continuous variable, [and] (g) the covarious matrices for each group must be approximately equal. (Sherry, 2006, p. 668)

The aforementioned assumptions were met in the present analysis. The assumption that the experimental and control groups exhibit multivariate normal distribution based on knowledge and attitude scores was met through a plot of Mahalanobis distances and paired chi-squares (Sherry, 2006). The homogeneity of variance assumption was not met as indicated by Box's $M[F(1,2)=26.176, p<.05]$. Box's $M$ is an especially sensitive test of nonnormality, and in the presence of large or relatively equal sample size between groups, DDA is robust even though the homogeneity assumption is not met (Sherry, 2006). Thus, the large total sample size of $N=212$ and relatively equal group sizes made DDA a robust analysis for this research question.

Summary of canonical discriminant functions is reported in Table 3. These results indicated that knowledge and attitude accounted for $44 \%$ of the differences between the two groups and reflected a large effect. We considered structure coefficients and standardized discriminant function coefficients to determine which of the two variablesknowledge or attitude-contributed to the larger group differences. 
Table 3 Wilks's Lambda and Canonical Correlation

\begin{tabular}{ccccccc}
\hline Function & $\begin{array}{c}\text { Wilks's } \\
\text { Lambda }\end{array}$ & $\mathbf{X}^{2}$ & df & $p$ & $R_{c}$ & $R_{c}{ }^{2}$ \\
\hline 1 & .6 & 121.2 & 2 & $<.001$ & .7 & $44 \%$ \\
\hline
\end{tabular}

Standardized coefficients depict simultaneous contribution of all variables and are limited in depicting the absolute contribution of any one variable (Sherry, 2006). In this study, knowledge $(.986 ; 100 \%)$ contributed more to creating the synthetic variable than attitude (.034; 18.7\%). The structure matrix depicts the relationship between the dependent variables and the function (Sherry, 2006). Furthermore, structure coefficients are Pearson $r$ statistics ranging from -1 to +1 and depict unique contribution of each variable to the synthetic dependent variable (Sherry, 2006). The structure matrix revealed that knowledge had the strongest correlation with the grouping variable (1.00), followed by attitude (.432). For function 1, knowledge was predominantly responsible for group differences with knowledge being moderately positively correlated with attitude.

Group "centroids provide an estimate of where each of these variables fall relative to each other" (Sherry, 2006, p. 676). In this analysis, the group centroid for experimental and control were respectively .934 and -.834. Difference in knowledge was higher for participants in the experimental group than in the control group. Based on results, the experimental group was higher on Function 1 than the control group, indicating that the experimental group was more knowledgeable about NDEs with more positively impacted attitudes about NDEs than was the control group.

For the secondary research question, we used Pearson productmoment correlation to assess the relationship between NDE knowledge and attitudes among the entire sample of 212 participants. Pretest results were $r=.458, p<.01$, indicating a positive, significant moderate relationship between knowledge and attitude with a small effect $(r 2=.210)$. Posttest results were $r=.678, p<.01$, indicating a positive, significant, strong relationship with a medium effect $(r 2=$ .460). Thus, NDE knowledge and attitude showed a consistent, substantial positive relationship to each other with small to medium practical significance. 


\section{Discussion}

In the current study, we sought to explore the degree to which differences in pre- and posttest scores on knowledge and attitudes about NDEs contributed to group differences between experimental group licensed counselors who completed an online education program about NDEs and control group licensed counselors who completed an online education program consisting of material unrelated to NDEs. Results indicated that both knowledge and attitudes increased significantly for experimental group counselors compared to control group counselors. Additionally, knowledge and attitude about NDEs were positively, significantly correlated at pretest and posttest with small to medium effects.

Counselors in the current study effectively increased their knowledge, and their attitudes were slightly impacted, through the online education program consisting of approximately 2.75 hours of instruction: a video and two Powerpoint-with-audio programs. Due to the statistically significant improvement of knowledge and attitudes in counselors who participated in the NDE online education program, the current study makes a unique contribution to the professional literature in the field of near-death studies. In their comprehensive, critical review of practical applications of NDE research, Foster, James, and Holden (2009) concluded that healthcare providers needed and wanted further education about NDEs, but no widely available program had existed to meet that need. This finding is similar to the current study; in response to a quantitative survey question, $88 \%$ of the total 212 participants reported a lack of formal education regarding NDEs. However, in qualitative feedback, participants shared that they were nevertheless interested in learning more about NDEs and found the topic valuable and relevant to their work with clients. Indeed, Young, Wiggins-Frame, and Cashwell (2007) also reported a similar finding in their study of counselors' competency to address spiritual concerns with clients; respondents in their study indicated a need for additional training, such as workshops, seminars, and educational programs. Therefore, counselors have expressed a professional interest for more information about NDEs as it relates to their clinical work with clients. Now an effective program is available to meet this need.

It is important to note that NDE-educated counselors in this study showed greater improvement in knowledge than in attitudes about NDEs. Because a relatively more positive attitude is essential to the role of counselors working with NDErs, as it relates directly to their 
ability to demonstrate a nonjudgmental and unbiased disposition towards their clients (ACA, 2014; Greyson, 2000), the finding in this study that attitude improved less than knowledge calls for speculation. One possible explanation may be inferred from previous literature regarding other healthcare professionals' knowledge and attitudes about NDEs. For example, Barnett (1991) surveyed nurses' knowledge and attitudes about NDEs and found that participants demonstrated positive attitudes about NDEs but only sufficient knowledge. Additionally, Cunico (2001) reported that the nurses in her study expressed positive attitudes about NDEs but only a modest amount of knowledge. Furthermore, Moore (1994) studied physicians' knowledge and attitudes towards NDEs and found positive attitudes about NDEs but only sufficient knowledge. Likewise, Betchel et al. (1992) found that the majority of clergy in their sample lacked accurate knowledge of NDEs and possessed moderately positive attitudes about NDEs. Moreover, Walker and Russell (1989) assessed psychologists' knowledge and attitudes towards NDEs and concluded that the psychologists exhibited moderately positive attitudes toward the topic but limited knowledge. Although the KANDES has not been standardized to provide scoring criteria for what constitute "sufficient" knowledge or "positive" attitude, counselors in the experimental condition may have shown relatively less improvement in attitudes because of a "ceiling effect": Their attitudes about NDEs may have already been relatively positive despite a relatively greater lack of knowledge about them.

Another possible explanation may come from research on the relationship between knowledge and attitude. Regarding multicultural competence, Arredondo et al. (1996) argued that there is a relationship between knowledge of a particular minority group and attitudes toward that same group. Indeed, Alderson, Orzeck, and McEwen (2009) surveyed 223 school counselors' knowledge and attitudes about gay males. The authors found that higher levels of knowledge predicted lower negative attitudes. Therefore, greater knowledge may lead people to develop more positive views toward a population. However, Farmer, Welfare, and Burge (2013) surveyed 468 counseling students, counselors, and counselor educators to assess their knowledge and attitudes about lesbian, gay, and bisexual clients. These authors found that participants reported positive and affirming attitudes but low knowledge in working with this population. Similar to the current study, Israel and Hackett (2004) compared the effects of informationbased and attitude-based interventions on counselor trainees' knowledge and attitudes toward lesbian, gay, and bisexual clients. Providing 
information yielded higher levels of knowledge; however, the exploration of attitude led to more negative attitudes. The authors suggested that in order to produce positive change in attitudes, participants may need more extensive training over a longer period of time (Israel \& Hackett, 2004). Therefore, it may befit counselors to engage in multiple trainings or follow-ups to allow for the appropriate amount of time and depth of reflection necessary for improved attitudes. Thus, an alternate possible explanation for the results of the current study is that experimental group counselors' attitudes improved less than their knowledge because of a "lag factor" between acquisition of knowledge and the effect of that acquisition on attitudes. Whether the finding of relatively less improvement in NDE attitudes compared to knowledge is best attributed to a ceiling effect, a lag effect, or some other factor remains for future researchers to determine.

Another important finding is the participants' experiences in completing the online education program. We calculated their unsolicited narrative responses and found that 85 of the 100 experimental group participants provided positive comments about the topic(s), content, and/or means of delivering the information. No participant expressed a negative comment about topic(s) or content. The only negative report was difficulty completing the program in one sitting. We had originally specified the one-sitting requirement to ensure that participants would not acquire information on NDEs from outside sources between pre- and post-test-so that results could be confidently attributed to the online program. However, due to low response rate, we later informed participants that they could complete the survey in multiple sittings as long as they did not seek educational material related to the study until after they submitted the post-test survey.

Numerous factors may have contributed substantially to the high attrition rate of participants who began but did not complete the studysuch as disinterest or aversion to the topic once it was revealed, length of the program, or technical difficulties with the online programs, the latter of which several participants reported. However, most salient to the focus of this article were the overall themes that seemed to emerge from experimental group participants: that they found the NDE educational program beneficial to their clinical practice, they enjoyed the interactive nature of the program-especially hearing NDE narratives in experiencers' own words, and they suggested topics of interest for future NDE program. Following is a representative list of these participants' narratives: 
- I believe this will strengthen my clinical skills and has shown me the another way that validation or client's thoughts, and experiences is beneficial in counseling. I hope that in the future to be able to correctly name a NDE and help the NDE'er to process their experience without doing any damage to the person. I never would have thought to do a CEU course on this topic or realize how important the knowledge is to health care professionals.

- I feel more equipped as a professional to discuss and provide education about NDEs if I encounter someone who has experienced one. I also have a resource to provide to individuals to help them seek extra support from people who have experienced something similar.

- NDE has never been mentioned/included in any of my formal education, nor CEU opportunities.

- I really enjoyed hearing the NDE's, as it allowed me to think about what I was hearing from a therapeutic perspective, and how I might respond to a client discussing that experience.

- I would add other experiences from other ethnicities with different culture background along with ages.

- Be nice to see you offer this across the nation for expanded CEUs.

- I had difficulty accessing the interactive module. Making it more accessible from a mobile device.

- Personally, I would like to know more about the experiences of people of other cultures and faiths ... the fact that they are similar across age, race, and spiritual orientation is more fascinating to me than our own Western/American perspectives.

- Put all the educational materials together into one package.

- Include more information on the negative NDE and how to help people who have these.

- I would suggest updating the accounts of near death experiences to include ones that occurred more recently.

- I believe this educational package needs to be made available to every health/mental healthcare provider; including clergy and family members of love ones who are terminally ill or have experienced a traumatic experience.

\section{Limitations and Future Research}

Despite the effectiveness of the NDE online education program on our counselor participants' knowledge and attitudes, several limitations in the current study exist, including limited geographic location of participants, convenience sampling, high attrition rate, low response rate, and an overrepresentation of White and female participants. Selection of only one state from each of the four ACA regions of the 
United Sates made possible a national study. However, it is important to consider that the counselors from the four representative states may not have represented all counselors from their respective regions; therefore, their responses-and, thus, the results of this study-may not be generalizable to all counselors nationwide. Additionally, race and sex distributions of participants were skewed in favor of White (83\%) and female (87.3\%). However, these percentages are fairly representative of the counseling field. According to the U.S. Census Bureau (2015), $70.4 \%$ of counselors are White, and $70.9 \%$ of counselors are female. Furthermore, Black or African American counselors represent $19.5 \%$ of all counselors; they comprise the second most common race/ ethnicity in the counseling field. Similarly, in the current study, Black or African American counselors were the second-highest represented racial/ethnic group. Therefore, the race and sex distributions of our participants were only somewhat more skewed than the distributions of counselors nationwide according to census data. Thus, the results may be considered reasonably representative with regard to race and gender diversity. However, future researchers will hopefully broaden their sample to additional geographical locations, which may lead to participant race and sex distribution even more closely mirroring that of the counseling field at large. In the meantime, generalizability of the results of this study to US counselors at large may be considered suggestive but not definitive.

Several participants in the current study reported technical difficulties in the process of accessing the three-part NDE program. Originators of the program are currently consulting with distance learning experts to seek a means to streamline program delivery to make it more usable for a wider audience. Failing this goal, they will create a tutorial explaining how to use the program, including screen shots of each step and a webpage of answers to frequently asked questions of a technical nature. To reduce the degree of technical difficulties for potential researchers and participants, the authors can offer suggestions for survey design in a future manuscript.

Future researchers could conduct a qualitative study to provide more enriching data. Participants could complete interviews or focus groups after completing this NDE online education program to better understand their perceptions and experiences related to knowledge and attitude. Other possible studies include replication of this study with providers and student providers from various other medical, mental, and spiritual/religious healthcare fields besides counseling. Additionally, researchers could examine client experiences related to 
counselors who have completed this NDE online education program. For example, clients who have experienced an NDE and an independent observer would rate their counseling sessions with counselors who had and had not completed the NDE training. Researchers would analyze and compare the ratings of the NDErs and the independent observers for both groups of counselors to determine whether, indeed, increased knowledge and improved attitudes translate into observable enhancement of counseling skills.

\section{Implications}

The results of this study carry implications for healthcare professional education. During NDEs, experiencers are typically thrust into a usually hyper-real experience of an alternate reality for which Western culture, at least, largely has not prepared them. Consequently, in the aftermath most NDErs need to psychospiritually process their experiences so that they can integrate their NDEs into their self-concepts, worldviews, and subsequent lives. When healthcare professionalsmedical, mental, and spiritual/religious-and significant others in NDErs' lives reject the experience, disapprove of it, or label it as mental illness, NDErs may suppress their experiences instead of gaining the potential positive benefits inherent in them (Noyes et al., 2009, p. 55). Furthermore, NDErs have reported that they found disclosure experiences to be negative when their NDE was not recognized as an NDE or was disbelieved, dismissed, diagnosed/pathologized, or demonized (Holden, 2012; Holden et al., 2014). Therefore, relatively more accurate knowledge and positive attitudes about NDEs may prevent client harm in a variety of healthcare settings.

Despite this likelihood, previous research has shown health professionals to be relatively lacking in knowledge about NDEs but interested in gaining such knowledge. Regarding medical healthcare professionals, Moore (1994) found that only $16 \%$ of a sample of 143 physicians possessed sufficient knowledge about NDEs. Additionally, researchers have stated that nurses could benefit from improved knowledge about NDEs (Barnett 1991; Cunico, 2001). Regarding mental health professionals besides counselors, Walker and Russell (1989) found that psychologists "demonstrated limited knowledge of near-death phenomena, but maintained a moderately positive attitude toward the topic" (Walker \& Russell, 1989, p. 109). Regarding spiritual/religious healthcare providers such as clergy and chaplains, Betchel et al. (1992) found that the majority of clergy in their sample lacked accurate knowledge 
of NDEs and expressed a willingness to participate in an NDE related educational programs due to the inherent religious and spiritual implications of the experience. Holden et al.'s (2014) more recent findings indicate that previous findings about healthcare professionals' relative lack of knowledge but desire for education persist. Participants in this current study reflected a similar profile-and also demonstrated that the NDE online education program largely fulfilled their desire.

Although the sample in the current study consisted only of counselors, it may behoove healthcare educators, supervisors, and continuing educators to provide their students and supervisees with the NDE online education program of current study in order to address this need. Because the program is available to a wide range of helping professionals in multiple settings, it could easily be included in the curricula for these professionals' education and/or continuing education.

Specifically with regard to counselor education, because of the spiritual aspect of NDEs (Ingersoll \& Zeitler, 2010; Marquis, 2012; Wilber, 2000), counselor educators could incorporate the NDE educational program into a multicultural counseling course, either by requiring its completion out-of-class and following up with in-class activities or by adapting it to a face-to-face format. In either case, a counselor educator may add more reflective and experiential processes to increase depth of knowledge and improved attitude. Counselor trainees may benefit from the addition of peer interaction in a cooperative learning environment, an important aspect of teaching multicultural competence (Dickson, Jepsen, \& Barbee, 2008). Furthermore, agencies, group practices, post-graduate counselor supervisors, universities, or continuing education providers could utilize the NDE online education program to improve supervisees' or professional counselors' knowledge and attitudes about NDEs and NDErs.

\section{Conclusion}

The life changing and often stressful aftereffects of NDEs motivate many NDErs to seek support from healthcare providers for the integration of their experiences (Greyson, 2000). When NDErs encounter a positive and supportive attitude rather than a negative, rejecting attitude, they internalize and integrate their NDEs more healthfully (Greyson \& Liester, 2004). Conversely, negative disclosure experiences can be harmful to NDErs. Counselors' knowledge and attitudes about NDEs appear to be critical variables in their ability to uphold the ethical imperative to do no harm to clients (ACA, 2014). However, some 
counseling professionals and students possess inaccurate knowledge about and negative attitudes about NDEs (Pace, 2013). Widely accessible education to improve knowledge and attitudes about NDEs may minimize harm and enhance the help available to NDErs.

The purpose of this study was to determine the impact of an online education program on counselors' knowledge and attitudes about NDEs. The statistically and practically significant findings between groups in this study suggest that counselors' knowledge and attitudes about NDEs could be reasonably expected to improve based upon their completion of a three-part online NDE educational program. Findings from this study demonstrate the NDE online education program's viability as an education option for counselors-in-preparation and a continuing education option for counselors. Due to the limitations previously discussed, it is important that future researchers replicate and extend this study to affirm its effectiveness with a wider sample of counselors, counselors-in-preparation, and other healthcare providers and providers-in-training and to affirm that increased knowledge and improved attitudes about NDEs translates into more effective healthcare treatment.

\section{References}

Alderson, K. G., Orzeck, T. L., \& McEwen, S. C. (2009). Alberta High School counsellors' knowledge of homosexuality and their attitudes toward gay males. Canadian Journal of Education, 32, 87-117.

American Counseling Association. (2014). 2014 ACA code of ethics. Retrieved from http://www.counseling.org/docs/ethics/2014-aca-code-of-ethics.pdf?sfvrsn=4.

American Counseling Association. (2016). Division, regions and branches. Re trievedfrom https://www.counseling.org/about-us/divisions-regions-and-branches.

Anderson, T. (2008). Toward a theory of online learning. In T. Anderson (Ed.), Theory and practice of online learning (pp. 15-44). Edmonton, AB: AU Press.

Arredondo, P., Toporek, R., Brown, S. P., Jones, J., Lock, D. C., Sanchez, J., \& Stadler, H. (1996). Operationalization of the multicultural counseling competencies. Journal of Multicultural Counseling \& Development, 24, 42-78.

Barnett, L. (1991). Hospice nurses' knowledge and attitudes toward the neardeath experience. Journal of Near-Death Studies, 9(4), 225-232. doi:10.17514/ JNDS-1991-9-4-p225-232.

Bechtel, L. J., Chen, A., Pierce, R. A., \& Walker, B. A. (1992). Assessment of clergy knowledge and attitudes toward near-death experiences. Journal of Near-Death Studies, 10(3), 161-170. doi:10.17514/jnds-1992-10-3-p161-170.

Benshoff, J. M., \& Gibbons, M. M. (2011). Bringing life to e-learning: Incorporating a synchronous approach to online teaching in counselor education. Professional Counselor, 1(1), 21-28. doi:10.15241/jmb.1.1.21.

Betz, N. E. (1987). Use of discriminant analysis in counseling psychology re- 
search. Journal of Counseling Psychology, 34, 393-403. doi:10.1037/002 2-0167.34.4.393.

Blackmore, C., Tantam, D., \& van Deurzen, E. (2006). The role of the Etutor Evaluating tutor input in a virtual learning community for psychotherapists and psychologists across Europe. International Journal of Psychotherapy, special edition, 10(2), 35-46.

Blackmore, C., van Deurzen, E., \& Tantam, D. (2005). A comparative evaluation of eLearning versus traditional 'face-to-face' teaching methods in a psychotherapy training program. In S. Dasgupta (Ed.), Encyclopedia of virtual communities and technologies (pp. 174-178). Washington, DC: George Washington University.

Broome, K. (Producer). (2002). The day I died: The mind, the brain, and neardeath experiences [Motion picture]. Glasgow, Scotland, UK: British Broadcasting Corporation. Available at https://topdocumentaryfilms.com/day-i-died/.

Bush, N. E. (2009). Distressing Western near-death experiences: Finding a way through the abyss. In J. M. Holden, B. Greyson, \& D. James (Eds.), The handbook of near-death experiences: Thirty years of investigation (pp. 63-86). Santa Barbara, CA: Praeger/ABC-CLIO.

Cohen, J. (1988). Statistical power analysis for the behavioral sciences (2nd ed.). Hillsdale, NJ: Lawrence Earlbaum Associates.

Cunico, (2001). Knowledge and attitudes of hospital nurses in Italy related to near-death experiences. Journal of Near-Death Studies, 20(1), 37-50. doi: 10.17514/jnds-2001-20-1-p37-50.

Dickson, G. L., Jepsen, D. A., \& Barbee, P. W. (2008). Exploring the relationships among multicultural training experiences and attitudes toward diversity among counseling students. Journal of Multicultural Counseling and Development, 36, 113-126.

Dominguez, P. S., \& Ridley, D. R. (2001). Assessing distance education courses and discipline differences in their effectiveness (1). Journal of Instructional Psychology, 28(1), 15.

Evans, J. D. (1996). Straightforward statistics for the behavioral sciences. Pacific Grove, CA: Brooks/Cole.

Farmer, L. B., Welfare, L. E., \& Burge, P. L. (2013). Counselor competence with lesbian, gay, and bisexual clients: Differences among practice settings. Journal of Multicultural Counseling and Development, 41(4), 194-209.

Fenwick, P., \& Fenwick, E. (1995). The truth in the light: An investigation of over 300 near-death experiences. London, UK: Headline.

Forester-Miller, H., \& Davis, T. E. (1995). A practitioner's guide to ethical decision making. Alexandria, VA: American Counseling Association.

Foster, R., James, D., \& Holden, J. M. (2009). Practical applications of neardeath experiences research. In J. M. Holden, B. Greyson, \& D. James (Eds.), The handbook of near-death experiences: Thirty years of investigation (pp. 235258). Santa Barbara, CA: Praeger/ABC-CLIO.

Greyson, B. (1997). The near-death experience as a focus of clinical attention. Journal of Nervous and Mental Disease, 185(5), 327-334. doi:10.1097/ 00005053-199705000-00007.

Greyson, B. (2000). Near-death experiences. In E. Cardeña, S. J. Lynn, \& S. Krippner (Eds.), Varieties of anomalous experience: Examining the scientific evidence (pp. 315-352). Washington, DC: American Psychological Association. 
Greyson, B. (2014). Near-death experiences. In E. Cardeña, S. J. Lynn, \& S. Krippner (Eds.), Varieties of anomalous experience: Examining the scientific evidence (2nd ed.; pp. 333-367). Washington, DC: American Psychological Association.

Greyson, B., \& Liester, M. B. (2004). Auditory hallucinations following neardeath experiences. Journal of Humanistic Psychology, 44(3), 320-336. doi: 10.1177/0022167804266281.

Greyson, B., Kelly, E. W., \& Kelly, E. F. (2009). Explanatory models for neardeath experiences. In J. M. Holden, B. Greyson, D. James, J. M. Holden, B. Greyson, D. James (Eds.), The handbook of near-death experiences: Thirty years of investigation (pp.213-234). Santa Barbara, CA: Praeger/ABC-CLIO

Groth-Marnat, G., \& Summers, R. (1998). Altered beliefs, attitudes, and behaviors following near-death experiences. Journal of Humanistic Psychology, 38(3), 110-125. doi:10.1177/00221678980383005.

Herlihy, B., \& Corey, G. (2006). ACA ethical standards casebook (6th ed.). Alexandria, VA: American Counseling Association.

Holden,J.M.(2008). Near-deathexperiences, part 1:Recognizingapleasurableneardeathexperience (online continuing education program for healthcare providers). Durham, NC: International Association for Near-Death Studies. Available at http://www.iandsce.org/NDE/Index.html.

Holden, J. M. (2012). After-math: Counting the aftereffects of potentially spiritually transformative experiences. Journal of Near-Death Studies, 31(2), 6578. doi: 10.17514/JNDS-2012-31-2-p65-78.

Holden, J. M. (2015). Avoiding harm: Responding therapeutically to disclosure of a near-death experience [PowerPoint presentation]. Available at https://www .youtube.com/watch?v=8u0Ia4BFcoA.

Holden, J. M. (2017). Near-death experiences. In R. D. Foster \& J. M. Holden (Eds.), Connecting soul, spirit, mind, and body (pp. 107-118). Denton, TX: University of North Texas Libraries. doi:10.12794/sps.connecting-008-3

Holden, J. M., Kinsey, L., \& Moore, T. R. (2014). Disclosing near-death experiences to professional healthcare providers and non-professionals. Spirituality in Clinical Practice, 1(4), 278-287. doi:10.1037/scp0000039.

Holden, J. M., Long, J., \& MacLurg, B. J. (2009). Characteristics of Western near-death experiencers. In J. M. Holden, B. Greyson, \& D. James (Eds.), The handbook of near-death experiences: Thirty years of investigation (pp. 109134). Santa Barbara, CA: Praeger/ABC-CLIO.

Holden, J. M., Oden, K., Kozlowski, K., \& Hayslip, B. (2011). Teaching about near-death experiences: The effectiveness of using The Day I Died. Omega: The Journal of Death and Dying, 63(4), 373-388.

Holden, R. R., Fekken, G. C., \& Cotton, D. H. G. (1991). Assessing psychopathology using structured test-item response latencies. Psychology Assessment, 3, 111-118. doi:10.1037/1040-3590.3.1.111.

Ingersoll, R., \& Zeitler, D. M. (2010). Integral psychotherapy: Inside out/outside in. Albany, NY: State University of New York Press.

Israel, T., \& Hackett, G. (2004). Counselor education on lesbian, gay, and bisexual issues: Comparing information and attitude exploration. Counselor Education and Supervision, 43(3), 179-191.

Kim, S., \& Sherry, A. (2010). Descriptive discriminant analysis. In N. J. Salkind 
(Ed.) The SAGE encyclopedia of social science research methods (pp. 349-352). New York, NY: SAGE.

Klecka, W. R. (1980). Discriminant analysis. Beverly Hills, CA: Sage.

Landis, J. R. \& Koch, G. C. (1977). The measurement of observer agreement for categorical data. Biometrics, 33(1), 159-174. doi:10.2307/2529310.

Lewis, J., \& Coursol, D. (2007). Addressing career issues online: Perceptions of counselor education professionals. Journal of Employment Counseling, 44(4), 146-153. doi:10.1002/j.2161-1920.2007.tb00033.x.

Marquis, A. (2012). The integral intake: A guide to comprehensive idiographic assessment in integral psychotherapy. New York, NY: Routledge.

McEvoy, M. D. (1990). The near-death experience: Implications for nursing education. Loss, Grief \& Care, 4(1-2), 51-55.

Means, B., Toyama, Y., Murphy, R., Bakia, M., \& Jones, K. (2009). Evaluation of evidence-based practices in online learning: A meta-analysis and review of online learning studies. Washington, DC: U.S. Department of Education.

Moody, R. A. (1975). Life after life. Harrisburg, PA: Stackpole.

Moore, L. H. (1994). An assessment of physicians' knowledge of and attitudes toward the near-death experience. Journal of Near-Death Studies, 13(2), 91107. doi: 10.17514/JNDS-1994-13-2-p91-102.

Noyes, R., Fenwick, P., Holden, J. M., \& Christian, S. R. (2009). Aftereffects of pleasurable Western adult near-death experiences. In J. M. Holden, B. Greyson, \& D. James (Eds.), The handbook of near-death experiences: Thirty years of investigation (pp. 41-62). Santa Barbara, CA: Praeger/ABC-CLIO.

Pace, L. (2013). Development of the knowledge and attitudes toward near-death experiences scale (Doctoral dissertation). Retrieved from UNT Digital Library. http://digital.library.unt.edu/ark:/67531/metadc500204/.

Pace, L., Holden, J. M., Blalock, S., Holliman, R., \& Henson, R. K. (2016). Development of the knowledge and attitudes about near-death experiences scale (KANDES). Journal of Near-Death Studies, 34(3), 173-188. doi:10.17514/ JNDS-2016-34-3-p173-188.

Roberta Moore Video Productions (Producer). (2013). Near-death experiences: What medical professionals need to know [Motion picture]. Fort Myers, FL: Blue Marble Films. Available at [https://vimeo.com/139014560].

Sheeler, R. D. (2005). Teaching near-death experiences to medical students. Journal of Near-Death Studies, 23(4), 239-247. doi:10.17514/JNDS-200523-4-p239-247.

Sherry, A. (2006). Discriminant analysis in counseling psychology research. Counseling Psychologist, 34(5), 661-683. doi:10.1177/0011000006287103.

Sidgwick, E. M. (1885). Notes on the evidence, collected by the society, for phantasms of the dead. Proceedings of the Society for Psychical Research, 3, 69-150.

Tabachnick, B. G., \& Fidell, L. S. (2007). Using multivariate statistics (5th ed.). New York, NY: HarperCollins.

Taylor, S., \& van Lommel, P. (2006). Pleasurable Western adult near-death experiences: Circumstances, contents, and incidence. [Video recording]. Retrieved from http://www.iands.org/conferences/2006_conference_presentations/.

U.S. Census Bureau. (2015). Data USA: Counselors. Retrieved from https:// datausa.io/profile/soc/211010/.

Walker, B. A., \& Russell, R. D. (1989). Assessing psychologists' knowledge and 
attitudes toward near-death phenomena. Journal of Near-Death Studies, 8(2), 103-110. doi:10.17514/JNDS-1989-8-2-p103-110.

Watson, P. (2016, April 15). Computing effect sizes. Retrieved from http:// imaging.mrc-cbu.cam.ac.uk/statswiki/FAQ/Escomp.

Wilber, K. (2000). Integral psychology: Consciousness, spirit, psychology, therapy. Boston, MA: Shambhala.

Young, J. S., Wiggins-Frame, M., \& Cashwell, C. S. (2007). Spirituality and counselor competence: A national survey of American Counseling Association members. Journal of Counseling and Development, 85, 47-53.

Zhao, Y, Lei, J., Yan, B., Lai, C, \& Tan, H. S. (2005). What makes the difference? A practical analysis of research on the effectiveness of distance education. Teachers College Record, 107(8), 1836-1884. doi:10.1111/j.1467-9620.2005.00544.x.

Zingrone, N. A., \& Alvarado, C. S. (2009). Pleasurable Western adult near-death experiences: Features, circumstances, and incidence. In J. M. Holden, B. Greyson, \& D. James (Eds.), The handbook of near-death experiences: Thirty years of investigation (pp. 17-40). Santa Barbara, CA: Praeger/ABC-CLIO. 